\title{
Poster Abstract: Reputation System for Ensuring Data Trustworthiness of Crowdsourced Weather Stations
}

\author{
Alexander B. Chen \\ Civil and Environmental \\ Engineering \\ University of Virginia \\ Charlottesville, Virginia \\ abc8fq@virginia.edu
}

\author{
Madhur Behl \\ Computer Science \\ University of Virginia \\ Charlottesville, Virginia \\ madhur.behl@virginia.edu
}

\author{
Jonathan L. Goodall \\ Civil and Environmental \\ Engineering \\ University of Virginia \\ Charlottesville, Virginia \\ goodall@virginia.edu
}

\begin{abstract}
Decision making in utilities, municipal, and energy companies depends on accurate and trustworthy weather information and predictions. Recently, crowdsourced personal weather stations (PWS) are being widely used to provide a higher spatial and temporal resolution of weather measurements. For instance, increasing attention is being paid to the potential of PWS data to improve flash-flood assessment and prediction. However, tools and methods to ensure the trustworthiness of the crowdsourced data in real-time are largely missing. In this paper, we present a Reputation System for Crowdsourced Rainfall Networks (RSCRN) to assign trust scores to personal weather stations in a region. Using real PWS data from the Weather Underground service in the high flood risk region of Norfolk, Virginia, we validate the performance and robustness of the proposed RSCRN. The proposed method is able to converge to a confident trust score for a PWS within 10-20 observations of installation and can robustly respond to any discrepancies in the data due to failure or malicious intent. We benchmark the performance of the proposed method with high-fidelity and trusted hydrological sensor data, which are usually expensive to install. Collectively, the results indicate that the trust score derived from the RSCRN can reflect the collective measure of trustworthiness to the PWS, ensuring both useful and trustworthy data for modeling and decision-making in the future.
\end{abstract}

\section{CCS CONCEPTS}

-Information systems $\rightarrow$ Sensor networks; • Human-centered computing $\rightarrow$ Reputation systems;

\section{KEYWORDS}

Crowdsourcing, Sensor Networks, Reputation System, Trust, Rainfall Networks

\section{PROBLEM DEFINITION}

The goal of RSCRN is to assign trust scores to each PWS using beta reputation system [2], based on the data it provides to the network. This trust score represents a collective measure

Permission to make digital or hard copies of part or all of this work for personal or classroom use is granted without fee provided that copies are not made or distributed for profit or commercial advantage and that copies bear this notice and the full citation on the first page. Copyrights for third-party components of this work must be honored. For all other uses, contact the owner/author(s).

BuildSys '18, November 7-8, 2018, Shenzen, China

(C) 2018 Copyright held by the owner/author(s).

ACM ISBN 978-1-4503-5951-1/18/11 . \$ 15.00

https://doi.org/10.1145/3276774.3281020

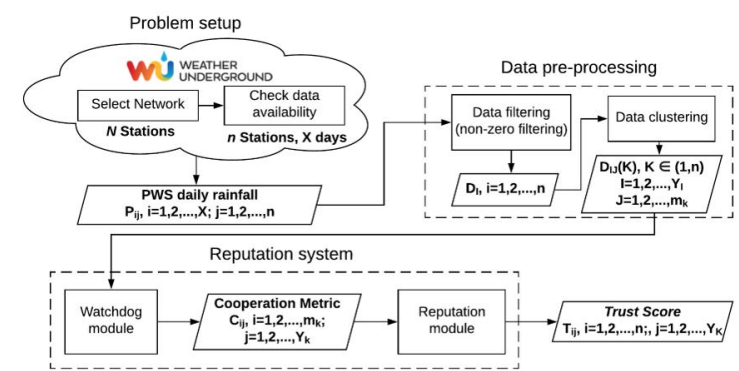

Figure 1: Overview of the RSCRN workflow: $N$ stations from a PWS network is first collected from the Weather Underground network; based on the data availability, the collected PWS daily rainfall data-set is then filtered and clustered into sub-data sets; the reputation system finally receives the sub-datasets and outputs trust score for each PWS in the network.

of trustworthiness of the station, which can be beneficial for decision-making and risk analysis in the future. The overview of the RSCRN workflow is shown in Figure 1.

\section{REPUTATION SYSTEM FOR CROWDSOURCED RAINFALL NETWORKS}

Reputation can be considered as a collective measure of trustworthiness (in the sense of reliability) based on the referrals or ratings from members in a community. In crowdsourced sensing applications, the period over which a user may contribute corrupted data may potentially be short-lived (particularly, when this happens unintentionally). It is desirable that the reputation scheme is responsive enough to capture such dynamic behavior.

In this paper, we implement a beta reputation system [2] for its advantage of simplicity, flexibility, and ability to counter most arbitrary sensor faults [1]. This beta reputation utilizes PWS data to assign trust scores to each station. The reputation system consists of two modules: (i)a watchdog module for detecting cooperative and non-cooperative behaviors of stations within the network, and (ii) a reputation module for managing and representing reputation information. 


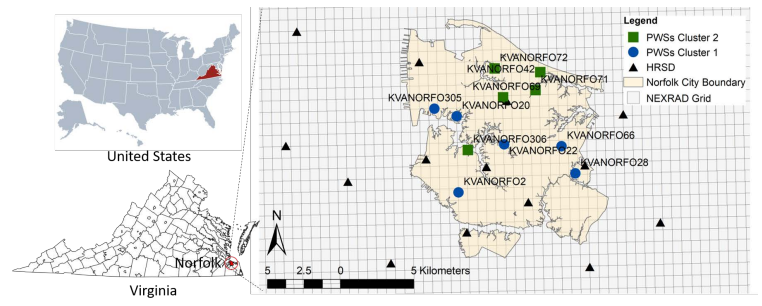

Figure 2: Available rainfall networks in the study area

\section{CASE STUDY}

\subsection{Study Area}

Norfolk, Virginia, USA was selected as the study area considering its growing vulnerability to flooding. Three sources of rainfall data, including one from crowdsourced weather networks and the other two from high-fidelity rainfall networks within and surrounding the the city of Norfolk are collected, as shown in Figure 2.

\subsection{Results and Discussion}

The results of the trust score evolution (Figure 3) show that the reputation system successfully identified the cooperative and non-cooperative behaviors of the PWSs over time, then gradually converge to a trust score after receiving 10-20 observations of rainfall measurements from the station. A simulation of including artificial station with faulty measurements to the network demonstrated the robustness of proposed the reputation method. The validation of the RSCRN, as shown in Figure 5, showed that the final trust score of PWSs are inversely proportional to the RMSEs of PWSs measurement and the ground-truth (defined as the bias-adjusted NEXRAD rainfall measurements). These analyses indicate that the trust score derived from the RSCRN can reflect the collective measure of trustworthiness to the PWS, ensuring highly spatial and temporal but also trustworthy data for general public, modelers and decision-makers.

In ongoing and future research, we aim to expand the proposed RSCRN to other regions with larger networks of PWSs. We also aim to enhance the applicability the RSCRN to general spatially distributed sensor networks, especially in locations where the ground-truth, or high-fidelity observation data is lacking. Ultimately, application-level benefits can be provided by taking the derived trust score of the RSCRN into account for hydrological and meteorological model simulation and prediction.

\section{ACKNOWLEDGMENTS}

This research is supported by the National Science Foundation under Grant No. CBET-1735587.

\section{REFERENCES}

[1] Saurabh Ganeriwal, Laura K Balzano, and Mani B Srivastava. 2008 Reputation-based framework for high integrity sensor networks. ACM Transactions on Sensor Networks (TOSN) 4, 3 (2008), 15

[2] Audun Josang and Roslan Ismail. 2002. The beta reputation system. In Proceedings of the 15th bled electronic commerce conference, Vol. 5. 2502 2511.

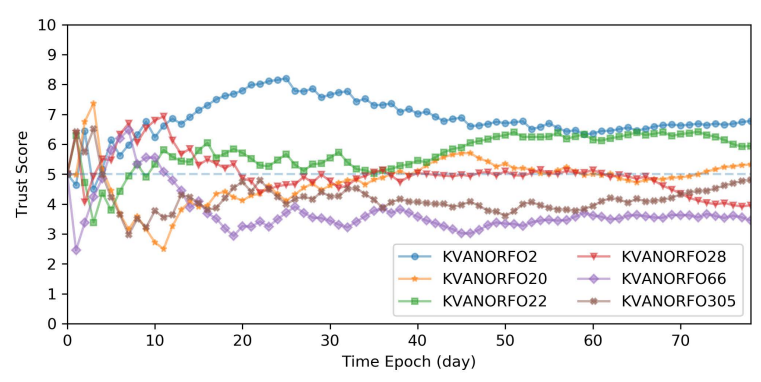

Figure 3: PWSs trust score evolution over time

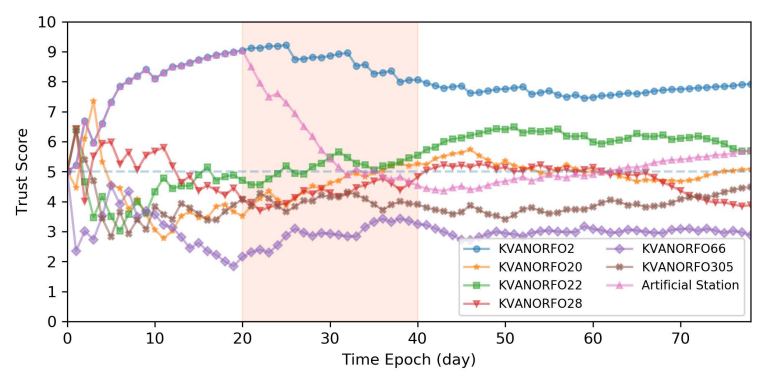

Figure 4: PWS trust score evolution over time with the introduction of a faulty artificial station: note that from epoch 20 to 40, the score of the artificial station (pink triangle) dropped significantly from a trust score 9.0 to a trust score of 4.5 because the non-cooperative behavior (due to the faulty measurements generated from the experiment) was detected starting from epoch 20 .

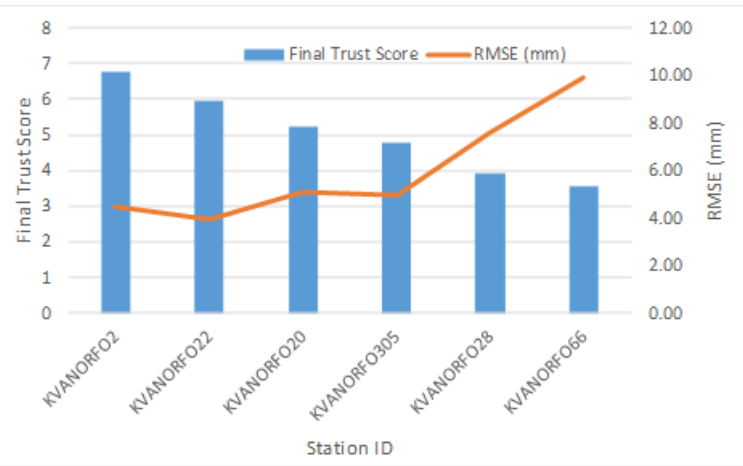

Figure 5: Final trust score derived from the RSCRN validating with the RMSE of PWS and bias-adjusted NEXRAD rainfall measurements 